\title{
Pour une approche génétique de la BD
}

\section{Pierre-Marc de Biasi}

\section{(2) OpenEdition}

\section{Journals}

\section{Édition électronique}

URL : https://journals.openedition.org/genesis/1700

DOI : 10.4000/genesis. 1700

ISSN : 2268-1590

\section{Éditeur :}

Presses universitaires de Paris Sorbonne (PUPS), Société internationale de génétique artistique littéraire et scientifique (SIGALES)

\section{Édition imprimée}

Date de publication : 12 décembre 2016

Pagination : 19-42

ISBN : 9791023105490

ISSN : 1167-5101

\section{Référence électronique}

Pierre-Marc de Biasi, « Pour une approche génétique de la BD », Genesis [En ligne], 43 | 2016, mis en ligne le 20 novembre 2017, consulté le 17 août 2022. URL : http://journals.openedition.org/genesis/ 1700 ; DOI : https://doi.org/10.4000/genesis. 1700 


\title{
Pour une génétique de la bande dessinée
}

\author{
Pierre-Marc de Biasi
}

$\mathrm{A}$

vec la bande dessinée, la critique génétique aborde un secteur particulièrement actif et significatif de la créativité contemporaine. Ce genre artistique qui touche désormais la plupart des cultures constitue un domaine éditorial en pleine expansion qui, bien qu'assez récent, se trouve déjà doté d'une richesse archivistique de premier plan en matière de traces génétiques et de dossiers documentaires. Envisager dans quelles conditions et avec quelles méthodes la création d'une bande dessinée peut devenir interprétable constitue donc aujourd'hui une nouvelle priorité pour les études de genèse.

En termes de processus créatifs, la bande dessinée se présente comme un mode d'expression composite dans lequel se trouvent engagées plusieurs caractéristiques propres à différents univers artistiques : les arts plastiques (dessin, estampes, peinture, photo, arts décoratifs, architecture), les arts de l'image en mouvement (lanterne magique, cinéma, vidéo), quelques aspects des arts du spectacle (théâtre, opéra, danse, performance) et bien sûr, désormais, les technologies numériques de l'image et les nouvelles applications qui s'y développent. Mais quelles que soient leurs contributions à l'originalité du «neuvième art», ces différentes logiques visuelles ne peuvent faire oublier que la BD est avant tout un art du récit, associant les ressources du dessin et du texte, pour réaliser des œuvres narratives qui partagent avec les textes littéraires la même finalité éditoriale : aboutir à une publication imprimée qui est elle-même destinée à être lue.

Cette prévalence du modèle éditorial laisse supposer que les grandes phases de la genèse, les fonctions opératoires du travail de création et les principaux processus d'élaboration de l'œuvre s'ordonnent, pour la bande dessinée, autour d'un axe, assez similaire à celui de la genèse littéraire, qui va de la préfiguration d'un projet scénaristique à sa réalisation écrite et dessinée, puis de sa finalisation prééditoriale à son édition (en périodique et/ou en album). Sans exclure les productions les plus anciennes et les plus contemporaines, ni oublier les nouveaux horizons numériques de la bande dessinée, je me propose de réfléchir aux conditions d'une approche génétique du genre en prenant pour exemples le travail d'Hergé et le corpus canonique de ses œuvres.

\section{Dessin, esquisse, croquis, ébauche}

Aux processus créatifs des arts plastiques, la BD emprunte évidemment toutes les 
finales ont été élaborées, au travail qui leur a donné naissance et aux traces que ce travail a pu laisser derrière lui : les archives dessinées de l'œuvre. En tant qu'outil de travail et de conception du graphiste, du peintre, du sculpteur ou de l'architecte, le dessin n'est donc qu'un terme générique derrière lequel se décline toute une terminologie habitée par une gamme assez étendue de significations distinctes : croquis, esquisse, ébauche, étude, étude d'ensemble, étude de détail, étude de composition, schéma, tracé, calque de report, calque correctif, crayon, crayonné, rendu, etc. Comme le «disegno» classique (dessin/dessein), le «schizzo» renaissant ou le «primo pensiero» de la tradition italienne, le dessin de BD est un instrument d'investigation et une médiation heuristique pour explorer, articuler et tester les formes et les significations d'une proposition graphique.

Une génétique de la $\mathrm{BD}$ implique donc, comme il conviendrait également de le faire pour tous les arts plastiques, que l'on définisse, de manière assez rigoureuse, les différentes fonctions opératoires auxquelles répondent ces différentes formes de dessin. Pour se démarquer des autres arts, le vocabulaire professionnel de la BD a choisi de donner à ces documents de genèse graphique le nom générique de «crayonnés» ou le terme anglais de «rough». Mais «crayonné» et «rough» sont aussi vagues que «dessin». Reste donc à distinguer dans ces-dits crayonnés ce qui relève plutôt du croquis (sur le motif ou d'imagination), de l'esquisse préliminaire, de l'ébauche, de l'étude d'ensemble ou de l'étude de détail intégrant les éléments d'un calque documentaire, etc. Tout comme il parait également indispensable, pour chacune de ces formes de dessin, de pouvoir statuer sur son objet, sa fonction et la place qu'elle occupe dans la succession des étapes qui vont de l'initialisation du projet à l'achèvement de l'œuvre. À quoi sert cette proposition graphique ? À quelle séquence génétique appartient-elle? Une approche génétique de la bande dessinée présuppose que la notion de «crayonné» soit décomposée en autant d'entités distinctes qu'il existe de fonctions opératoires prises en charge par l'acte de dessiner.

\section{Architecture}

Indissociablement outil de pensée, méthode de travail et œuvre aboutie, le dessin de BD partage avec le dessin de l'architecte une vocation à construire et déployer une fiction d'espace en mobilisant les structures fondamentales de la représentation qui s'imposent le plus spontanément à notre croyance et à notre regard. En termes de figuration, le dessinateur de $\mathrm{BD}$ joue beaucoup à l'architecte, surtout lorsqu'il doit inventer de toutes pièces les espaces qu'il représente : dans les histoires d'anticipation ou de science-fiction pour les lieux sans précédent dans lesquels se déroule l'action (villes, monuments, espaces publics, habitats, etc.) qui doivent être représentatifs de cette période inconnue du futur. C'est l'architecture qui exprime le mieux et le plus directement la culture, les techniques et les valeurs de la société constituant le cadre et souvent aussi l'objet central du récit.

Mais le dessin d'architecture n'est pas moins essentiel quand il s'agit d'évoquer un passé lointain (l'Antiquité gréco-latine de Jacques Martin, dans Alix, par exemple) ou proche (la guerre de 1914-1918 dans la saga Adèle Blanc-Sec par Jacques Tardi). Au risque du cliché et du stéréotype, le dessin d'architecture est un topos sémiotique de la BD : c'est le château fort qui fait le médiéval, comme c'est la tour Eiffel ou le Sacré-Cœur qui fait Paris. Pour dessiner les architectures, référentielles ou imaginaires, le dessinateur se sert de ses propres croquis de repérages ou de photos dont le cadrage et le point de vue se combinent 
avec la tradition graphique du dessin d'architecte. Perspective, perspective cavalière, point de fuite, élévation, plan, axonométrie, etc. : c'est avec les ressources géométriques de l'architecte que le dessinateur bâtit la fiction référentielle d'un paysage, d'une rue ou d'un environnement urbain, la lisibilité d'un décor, la simulation d'un objet en trois dimensions, etc. Mais à la panoplie géométrale de l'architecte, l'image de bande dessinée ajoute le principe dynamique d'une image en mouvement, c'est-à-dire les ressources du cinéma (voir plus loin).

\section{L'estampe et la reproduction en nombre}

Le dessin de bande dessinée ne peut pas s'interpréter dans l'unicité autographique du dessin de peintre ou d'architecte : ce qu'il vise, c'est une performance graphique capable de jouer le rôle de matrice pour une reproduction en nombre. Et c'est ce qui rend le dessin de $\mathrm{BD}$ comparable, par certains aspects, au régime d'élaboration graphique propre à l'estampe. En termes d'anticipation de l'image finale qu'il s'agit de rendre publiable, le dessinateur de BD est à la fois le «pictor» (autographe) et le «sculptor» (allographe) de la planche gravée : il sait qu'à l'échelle des détails les plus fins de son dessin, comme à l'échelle des effets de matières qu'il met en œuvre (papier, encre, plume, lavis, rehauts, etc.), le passage à l'épreuve imprimée puis à l'impression en nombre ne se réalisera pas sans un minimum de déperditions qui peuvent aller de l'affaiblissement à la dégradation.

Son geste créatif doit donc porter au-delà de la réalisation du dessin lui-même jusqu'à une certaine maîtrise anticipée du processus de fabrication : en réalisant la planche originale, le dessinateur doit prévoir la reproductibilité intégrale et optimale de tous ses tracés, en s'assurant de leur stabilité et de leur résilience lors de leur conversion imprimée, en sachant simplifier et aller à l'essentiel, en sacrifiant tout ce qui, dans la subtilité et l'infinitésimal d'un graphisme, risque de devenir incontrôlable au cours des étapes ultérieures.

Ce que l'on a appelé la «ligne claire» est un style qui relève à la fois d'un minimalisme formel inspiré du modernisme, d'une certaine conception orientale de l'énergie du trait, et d'une économie favorisant la lisibilité, mais c'est aussi une procédure de précaution à l'égard des transitions toujours incertaines entre l'autographique, l'allographique et la reproduction en nombre à partir d'une matrice. Dans la $\mathrm{BD}$ traditionnelle, s'y ajoute la séparation technique entre les tracés linéaires et l'application des couleurs. Si le traitement des ombres peut, comme dans la gravure et la «manière noire», bénéficier de toutes les ressources autographiques de la hachure et de l'épaisseur de trait, la colorisation proprement dite relève quant à elle d'une étape postérieure généralement prise en charge par des intervenants spécialisés (coloristes et imprimeurs).

\section{Photo}

La bande dessinée est née en Suisse avec Töpffer dans les années 1827-1840 à une époque qui est aussi celle de l'invention en France de la photographie. Ces deux techniques, qui vont s'imposer l'une et l'autre comme de nouveaux médiums d'expression artistique, inaugurent une ère démocratique de l'image : grâce au papier industriel (pâte de bois et machines à papier) qui fait baisser le coût des journaux et grâce aux perfectionnements techniques des méthodes d'impression (rotatives) qui permettent un développement vertigineux de la presse illustrée, l'image (photogravure) devient un produit de consommation de masse.

Mais la photo joue aussi un rôle important dans la genèse même de la bande dessinée : outre que l'auteur-scénariste travaille souvent en accumulant un important dossier d'images 
photographiques pour documenter sa recherche et son écriture, la photo est avec le croquis à main levée, un médium essentiel du repérage et de la documentation iconographique par lesquels le dessinateur et son équipe graphique intègrent au dessin original des planches les éléments exogénétiques d'une iconographie référentielle.

\section{Arts décoratifs, design}

Liée à la presse, la BD est un mode d'expression particulièrement sensible à l'évolution des formes, des goûts et des tendances qui caractérisent l'esprit du temps. Mise en page, caractères typographiques, structuration de l'espace, charte graphique, style de tracé, gammes de couleurs : la BD rassemble de manière aussi spontanée que systématique et emblématique les caractéristiques stylistiques de son époque. Little Nemo in Slumberland de l'américain Winsor McCay, publié par l'hebdomadaire New York Herald puis par New York American entre 1905 et 1914, est une création visionnaire et un chef-d'œuvre du genre, mais constitue aussi une illustration parfaite des rêveries de formes et d'espace propres à l'Art nouveau. Barbarella, publiée dans V Magazine en 1962 et en album chez Éric Losfeld en 1964 (Le Terrain vague) est considérée comme la première bande dessinée pour adultes et anticipe sur les mouvements de libération sexuelle de 1968, mais c'est aussi, du point de vue du design et des arts décoratifs, un véritable manifeste du pop art.

\section{Arts de la scène et de l'image en mouvement}

Née au XIX ${ }^{\mathrm{e}}$ siècle, la BD n'est pas sans relation avec les technologies artistiques de l'époque, comme le slow art du «tableau vivant» et du «panorama», les séquences d'images peintes de la «lanterne magique», et les décompositions du mouvement par l'image photographique. Comme le théâtre (et en particulier comme le mélodrame romantique), le music-hall, la BD repose sur une mise en scène de personnages privilégiant l'action, les rebondissements et le mouvement. Mais c'est évidemment le cinéma qui constitue la référence artistique majeure : en termes d'esthétique comme en termes de praxis et de vocabulaire professionnel. La bande dessinée emprunte au cinéma une bonne partie de sa terminologie technique (scénario, synopsis, storyboard, séquencier, script, etc.) et cet emprunt recouvre de réelles similitudes de démarche qui vont se retrouver dans la description des phases génétiques de la création. Mais surtout, en faisant de l'image fixe dessinée le matériau paradoxal d'un art qui cherche à simuler l'image en mouvement, la bande dessinée emprunte au cinéma tout un arsenal de moyens visuels relatifs à la prise de vue qui ont été élaborés, théorisés et expérimentés par le septième art.

Qu'il s'agisse de la notion de champ (champ, contrechamp, hors-champ) et de profondeur de champ (premier, deuxième plan, arrière-plan), de la notion de plan (plans de décor : panoramique, général, de petit ensemble; plans de personnages : moyen, américain, rapproché, gros plan, très gros plan), de l'idée d'orientation des personnages (face, dos, profil droit ou gauche, trois quarts droit ou gauche) ou encore du principe des angles de vue (frontal, en perspective, plongée, contre-plongée, etc.), la syntaxe visuelle de la bande dessinée se situe délibérément dans le prolongement de la prise de vue cinématographique. Même constat en ce qui concerne le choix des mouvements (travelling latéral, tirets et lignes), le traitement des enchaînements entre les vignettes (décomposition d'un même mouvement, champ-contrechamp, zoom avant ou arrière, ellipse, flash-back), la dialectique de l'œil caméra objectif-subjectif 
(focalisation interne) et, bien entendu, les procédures essentielles du montage (chronologique, par retour en arrière - flash-back, analepse -, ellipse, en parallèle, en alterné, cut, raccords, fondus, leitmotiv). Au total, à cette différence près qu'il n'utilise pas de caméra et ne cadre que des objets imaginaires, c'est bien en tant que chef opérateur que le dessinateur de bande dessinée, aidé de son complice le scénariste, se saisit de son objet graphique pour introduire dans l'image fixe le miracle du mouvement. Pour parodier Malraux, on pourrait dire que la bande dessinée, c'est l'irruption du cinéma dans les arts graphiques.

Ces multiples logiques visuelles sont bien présentes dans ce qui fait l'originalité esthétique de la BD comme mode d'expression plastique, mais d'un point de vue génétique, les archives de la création obligent à considérer que la bande dessinée se présente avant tout comme une œuvre narrative, alliant les ressources du dessin et du texte, conçue comme un projet dont l'aboutissement normal est une édition imprimée en nombre destinée à être «lue», et dont la diffusion repose sur la chaîne graphique du journal et/ou du livre. Traditionnellement publiée et diffusée en périodique dans la presse (quotidien, revue, magazine) avant d'être imprimée sous la forme d'albums, le statut éditorial de la bande dessinée ressemble en tout point à celui du roman ou de la nouvelle du XIXe siècle qui faisait toujours l'objet d'une prépublication sous la forme de plusieurs livraisons en périodique avant de paraître en volume comme ouvrage chez un éditeur.

\section{Double ou simple genèse}

À la différence du projet de sculpture ou d'architecture qui fait du dessin le médium d'une première étape conceptuelle dont le rôle instrumental est de rendre possible le passage à une seconde genèse qui correspond à la phase concrète du chantier, c'est-à-dire à une logique constructive, ou à la différence de la genèse picturale qui utilise l'outil graphique pour préparer la main du peintre à cette seconde genèse que sera la réalisation proprement dite du tableau avec d'autres moyens que le dessin, l'auteur de bande dessinée, quant à lui, ne se trouve pas confronté à l'exigence d'une double genèse, ni à un changement de médium, ni à une séparation stricte entre préparation et réalisation. Son parcours graphique peut être complexe et traversé de multiples repentirs et remaniements, il n'en reste pas moins qu'il se trouve, comme l'écrivain, installé dans le continuum génétique d'un processus à la fois homogène, progressif et cumulatif qui est d'emblée celui de l'œuvre à l'état naissant.

Il n'y a donc pas deux genèses mais une seule : au fur et à mesure que le dessinateur et le scénariste progressent, séquence par séquence, dans l'interprétation scriptographique du scénario initial, ils progressent aussi vers l'aboutissement effectif de leur travail. Chaque dessin réussi peut être considéré à la fois comme une étape vers l'œuvre et comme un élément de son achèvement. En termes de matériau comme en termes d'élaboration, le premier brouillon de la première page d'un roman est déjà de la même nature - scripturale - que le texte final qui prendra place dans l'œuvre achevée. De même, fût-elle très éloignée du résultat final qui sera publié, la première planche crayonnée par le dessinateur de BD relève d'un processus de réalisation qui va procéder par essais, reprises, corrections et perfectionnements mais sans rupture : non seulement la planche répond aux prescriptions d'un scénario (texte) et d'un découpage (images) qui en fixent les contenus et qui, en principe, continueront à s'imposer

\section{Prévalence du modèle littéraire et éditorial}


d'un bout à l'autre de la genèse, mais surtout, les métamorphoses imprévisibles que pourront connaître les dialogues, les tracés et la mise en page de cette planche auront toujours lieu en respectant les limites matérielles et les ressources techniques d'un seul et même médium qui est précisément le couple scriptographique dessin-texte.

\section{Représentations tabulaires de la genèse}

Comme pour la création littéraire ${ }^{1}$, artistique ${ }^{2}$ ou scientifique ${ }^{3}$, on peut schématiser la genèse d'une œuvre de bande dessinée sous la forme d'un tableau à double entrée qui permet de distribuer plusieurs rubriques de contenus paradigmatiques sur l'axe syntagmatique d'une chronologie. L'orientation temporelle (d'un avant vers un après) s'inscrit verticalement de haut en bas (comme pour la lecture d'un texte) tandis que l'axe horizontal sert à décliner les caractéristiques de chaque phase en termes de processus, de fonctions et d'actions rapportés aux documents de travail qui en témoignent. Pour simplifier la lisibilité de cette représentation tabulaire et pour bien marquer les temps forts de la création, j'ai choisi de traiter la successivité globale des opérations de genèse en quatre moments : la préparation, la réalisation, la finalisation et l'édition.

Je propose donc cinq tableaux synthétisant les six grandes phases de ce type de genèse, depuis la recherche initiale de l'idée jusqu'aux rééditions posthumes de l'album. Le premier tableau est consacré aux deux phases préliminaires (exploration, initialisation) qui aboutissent à la rédaction du scénario. Le deuxième tableau s'attache à la phase centrale de la genèse, c'est-à-dire à la réalisation proprement dite des crayonnés, du séquencier programmatique au storyboard récapitulatif. Le troisième tableau est focalisé sur la phase de finalisation au cours de laquelle la réalisation de l'œuvre entre dans une logique prééditoriale, du crayonné prédéfinitif à la colorisation. Enfin, les quatrième et cinquième tableaux développent les deux dernières phases : le processus d'édition en périodique et/ou en album, de la préparation du film pour l'impression et du bon à tirer jusqu'aux rééditions posthumes de l'œuvre.

\section{Phase 1 : exploration}

\section{La naissance de «l'idée»}

Dans la phase préliminaire des recherches exploratoires où il s'agit de déterminer, au sens kantien du terme, «l'idée », c'est-à-dire la thématique et l'orientation générales du récit à venir, chaque auteur de bande dessinée (comme chaque créateur, en pareilles circonstances) possède sa propre méthode et ses propres rituels, sans d'ailleurs pouvoir toujours expliciter très clairement, dans l'après-coup, le détail de sa démarche et de son cheminement : un fait divers, le contexte sociohistorique et géopolitique, un désir ou une émotion, un film, une chose vue, un livre lu, un rêve, un événement autobiographique, etc. peuvent être déclencheurs d'un préprojet. Mais la recherche du nouveau «sujet» peut aussi, pour l'artiste, se formuler en termes de défi et de réflexion sur le médium lui-même et sur les évolutions formelles

1. Pierre-Marc de Biasi, Génétique des textes, Paris, CNRS Éditions, coll. « Biblis », 2011.

2. La Fabrique du titre. Nommer les œuvres d'art, dir. Pierre-Marc de Biasi, Marianne Jakobi et Ségolène Le Men, Paris, CNRS Éditions, 2012.

3. Pierre-Marc de Biasi, «Sciences : des archives à la genèse. Pour une contribution de la génétique des textes à l'histoire des sciences », Genesis, n² 20, «Écriture scientifique», 2003, p. 19-52. 
du genre : le désir de rivaliser avec une œuvre retentissante qui vient de paraître, le besoin de chercher des formes nouvelles, de renouveler son propre vocabulaire graphique, de surprendre le public par l'émergence d'un univers imaginaire inédit.

\section{Conditions préalables de la recherche}

À ce stade préliminaire, le processus d'invention qui paraît jouir de la plus complète liberté, est en fait très dépendant des objectifs que se fixent les auteurs, de leurs antécédents et du contexte dans lequel ils entendent développer leur projet. L'auteur (ou le binôme auctorial : dessinateur, scénariste) peut réfléchir aux contenus d'un nouvel épisode s'inscrivant dans une série qui compte déjà un ou plusieurs récits publiés. La recherche préliminaire est alors fortement programmée par le préexistant (genre narratif, chronologie relative des épisodes, héros principaux, personnages récurrents, lieux et époque de référence, thématiques déjà traitées, etc.) et souvent par un contrat qui lie l'auteur à un journal ou à un éditeur pour tous les développements à venir de la même série.

L'auteur peut aussi se lancer dans la recherche d'une nouvelle proposition narrative purement spontanée, qui va se définir sans aucune contrainte, au plus près du désir et de l'imaginaire des concepteurs. Mais même dans un tel cas de «recherche libre», il est rare que l'orientation générale des premières investigations puisse se profiler sans anticiper un cadre éditorial dans lequel le projet pourra se concrétiser, le moment venu, en termes d'édition, de fabrication et de diffusion. Une telle prévision est à vrai dire indispensable, ne serait-ce que pour savoir s'il s'agit de réfléchir à un projet de publication séquentielle en périodique ou au projet d'une histoire complète qui devra paraître en album.

Le projet d'un nouveau récit intégral en album laisse aux auteurs la même liberté que celle dont dispose un écrivain : soit réaliser le projet à leur rythme, en allant jusqu'à la phase 4 de finalisation, puis s'enquérir d'un éditeur qui consentira à publier l'album (moyennant éventuellement quelques modifications), soit rechercher d'emblée un éditeur pour obtenir un contrat et une programmation (ou un engagement), moyennant une présentation approfondie du projet (pitch) et un dossier convaincant qui devra contenir le scénario complet de l'histoire et au moins quelques planches abouties. La négociation avec l'éditeur ne pourra donc avoir lieu qu'à la fin des deux premières phases de la genèse, ou même, s'il s'agit de fournir à titre probatoire des séquences abouties, vers le milieu de la phase 3 (réalisation).

Le projet en périodique présuppose la collaboration avec un journal et fera également l'objet d'un minimum de négociation initiale avec la rédaction en chef qui pourra, comme l'éditeur, demander à juger sur pièces (pitch, synopsis, quelques crayonnés probatoires, croquis de quelques personnages et décors clés) ou qui acceptera de s'engager (et éventuellement de passer contrat et commande) après une simple discussion si les auteurs sont suffisamment reconnus dans le métier. Après accord de principe sur les contenus, l'esprit, le style et l'orientation générique (humour, aventure, science-fiction, récit historique, policier, etc.), les auteurs devront se conformer au format du périodique, aux propositions de calibrage et de maquette (par livraison : une bande, une planche, une double page ou une séquence) et, bien entendu, au rythme - quotidien, hebdo, mensuel - de la publication.

Dans ce cas la genèse sera séquentielle et, s'il s'agit d'un récit continu, le schéma initial de l'histoire (qui aura été présenté au journal pour acceptation) pourra, selon les cas, ou être respecté sans modification, ou au contraire connaître des remaniements au fur et à mesure 
des livraisons. La périodicité reproduit la situation classique du roman-feuilleton du XIXe siècle qui offrait aux lecteurs la possibilité d'influer sur l'évolution narrative et aux auteurs la liberté de surprendre le public par des rebondissements inattendus ou des clins d'œil à l'actualité immédiate. Le courrier des lecteurs a longtemps servi à ce type de feed-back dans la bande dessinée publiée en journal, mais l'édition numérique ne fait aujourd'hui qu'intensifier les possibilités d'interaction entre les créateurs et leurs publics.

Au total, qu'il s'agisse d'un projet pour périodique ou d'un album à éditer, les contraintes initiales sont donc nombreuses et assez puissantes pour s'imposer comme des cadres préliminaires orientant fortement la réflexion des auteurs au cours des deux premières phases de la genèse. Des présupposés établis et très détaillés qui orientent la formulation d'un nouveau thème pour une série déjà existante, aux cadres plus ouverts qui circonscrivent la recherche d'une idée sans antécédent, les phases d'exploration et d'initialisation du projet se caractérisent par un système assez serré de conditionnements : c'est dans un tel contexte préstructuré que l'exploration initiale (phase 1) pourra aboutir à une véritable initialisation (phase 2) sous l'effet des échanges entre l'auteur et les différents partenaires du projet.

Comme pour certains écrivains (Flaubert débattant de son prochain projet de roman avec ses amis Bouilhet, Du Camp, les frères Goncourt, Sand et avec son éditeur), certains architectes (les discussions préliminaires sur le programme et le préprojet, en réunions d'agence) et certains cinéastes (les séances de travail entre réalisateur, producteur et scénariste), cette phase exploratoire, pour l'auteur de bande dessinée, se traduit souvent et longtemps par de nombreuses concertations avec différents interlocuteurs (éditeur, amis, artistes, experts, etc.) mais commence presque toujours par de longues discussions de l'auteur avec lui-même : en régime monologique lorsque l'auteur est une seule et même personne (comme dans le cas d'Hergé) ou, plus souvent, en régime dialogique lorsque le dispositif de création est fondé sur la collaboration étroite entre un «littéraire s scénariste, responsable de l'imaginaire écrit, et un plasticien dessinateur, spécialiste de la représentation graphique. Dans les dispositifs plus industrialisés où l'œuvre en tant que produit éditorial se trouve prédéfinie de manière précise, les discussions peuvent aussi donner une place importante au "producteur-éditeur» et aux partenaires techniques chargés de la chaîne de production graphique.

\section{Endogenèse et exogenèse exploratoires}

Dans tous les cas de figure, cette première phase exploratoire se traduit par d'intenses allers et retours entre des recherches d'informations exogénétiques, où il s'agit pour les auteurs de se doter d'une riche documentation provisionnelle (lectures, enquêtes, repérages, etc.) et des recherches de formulation où le travail consiste pour eux à préfigurer (graphiquement et surtout littérairement) une histoire qui servira de base à l'ensemble de l'œuvre. Cette préfiguration est endogénétique (elle ne repose que sur la faculté de l'imagination à créer de toutes pièces une fiction), mais elle est aussi immédiatement concernée par la dialectique de l'exogenèse : les sources et les documentations extérieures sont destinées à être intériorisées par le projet au point de devenir la matière même de la fiction.

Une fois définie l'idée générale, les auteurs accumulent souvent d'importants dossiers de documentation préliminaire («tout» sur le sujet) autant pour s'informer techniquement sur l'univers référentiel du récit que pour nourrir leur imaginaire et relancer leur désir créatif. Mais en termes de processus, toutes les enquêtes et recherches documentaires (écrites 
et visuelles) opérées dans cette phase 1 se trouvent d'emblée orientées par un objectif prioritaire : l'initialisation du projet sous la forme d'un texte de fiction narrative originale. L'exogenèse exploratoire n'a donc pour vocation finale que de rendre possible et d'enrichir l'élaboration d'un document endogénétique rédigé par le scénariste du projet. Au cours de la phase 1, les enquêtes provisionnelles et les discussions entre dessinateur et scénariste peuvent avoir donné lieu à la rédaction préliminaire de quelques ébauches narratives sous la forme embryonnaire de plans, de canevas ou de trames. Mais c'est avec l'apparition de documents plus aboutis comme le «synopsis» et surtout le «scénario» proprement dit que le projet passe de la phase exploratoire à la phase d'initialisation.

Pour Hergé qui avait opté pour une concentration presque exclusive des moyens entre ses propres mains, et qui, par conséquent, dans cette phase, devait dialoguer surtout avec lui-même, le processus d'émergence de «l'idée » relève de la boîte noire personnelle et reste difficile à élucider :

La difficulté est de trouver une idée, un fil d'Ariane, un film très clair... autrement dit un scénario très simple! Je pars de toutes sortes de sujets, jusqu'à ce qu'il apparaisse. Comment? Je ne sais pas. Tout phénomène de création est difficile à définir. Ce que je sais, c'est que cela peut durer longtemps 4

Même si Hergé n'est pas très prolixe sur ses procédés inventifs, et conclut sur une sorte de déni d'élucidation, on peut s'arrêter avec profit sur les images et les notions qui lui viennent instinctivement à l'esprit, au cours de l'interview, pour caractériser cette errance des commencements. Selon lui, le cheminement vers «l'idée » suppose que l'on trouve le «fil d'Ariane»: c'est un cliché, certes, mais qui, pris au pied de la lettre, nous dit peut-être quelque chose d'important sur sa démarche exploratoire, sur ce travail de recherche initiale plus ou moins ritualisée. Chercher l'idée, c'est se risquer, comme Thésée, dans les couloirs les plus secrets et les plus obscurs du Labyrinthe : être seul, plonger dans un inconnu nocturne, aller au-devant de ses démons personnels au risque d'un face-à-face central avec son propre Minotaure. Pas de recherche ni de trouvaille sans un minimum de détour risqué par l'inconscient.

Mais trouver l'idée suppose d'être entré dans ce labyrinthe muni du protocole qui évitera d'y tourner en rond, en dévidant derrière soi le fil qui permettra d'en sortir : le fil (ou le «film», reprend immédiatement Hergé, par une sorte d'apposition en forme de lapsus) de la clarté, un «film très clair» précise-t-il. Pour un créateur rendu célèbre de son vivant par sa fameuse esthétique de «la ligne claire», cette épithète «clair», associée à l'idée hybride d'un fil qui serait un film, nous renvoie tout à la fois à l'image animée (l'enchaînement d'images qu'est le cinéma), à sa matière première (le cadrage de l'image photographique - «écriture de la lumière » qui en constitue le paradigme) et à l'exigence de «clarté », le tout se résumant pour Hergé par la découverte d'un «scénario très simple».

La fameuse «idée» qu'il s'agit de trouver est donc une syntagmatique narrative d'images (la concaténation d'un récit réduit à quelques paradigmes visuels élémentaires) dont la productivité inventive est proportionnelle à la simplicité. De quelle nature est ce «scénario»? Comment se forme-t-il dans l'esprit de son créateur? De quoi est-il composé ?

4. Hergé, interview parue dans La Libre Belgique, 30 décembre 1975. 


\section{Phases 1 et 2 : exploration, initialisation}

\section{Canevas, synopsis, scénario}

Schéma, maquette, plan, ébauche, canevas : les esquisses écrites les plus diverses peuvent naître au cours de la phase 1 d'initialisation à la faveur des discussions entre dessinateur et scénariste, mais c'est avec le «synopsis» que se fixent pour la première fois les lignes principales de l'histoire. Hergé parle pour sa part d'un «schéma de scénario » qui tiendrait le milieu entre le canevas et le synopsis, et qui jouerait un rôle de pense-bête pour la période qui précède la rédaction du scénario :

Quand je tiens mon thème, j'établis un schéma de scénario très rudimentaire : une vingtaine de lignes seulement, car, en fait, je l'ai en tête ${ }^{5}$.

Bref résumé anticipatif qui énonce les grandes articulations de la fiction, son contexte, ses personnages et ses principaux rebondissements, le synopsis désigne, sur le modèle du cinéma, le texte très succinct qui expose de manière schématique l'intention des auteurs et les contenus du futur scénario avec une présentation sommaire du sujet traité, des personnages et de l'évolution du récit. Si le synopsis, très synthétique, dépasse rarement deux ou trois pages, le scénario d'une bande dessinée, plus resserré qu'au cinéma, pourra compter, selon les cas, entre une dizaine et une cinquantaine de pages. Rédigé par le scénariste qui le conçoit et le formule en tenant compte de le figurabilité de chaque élément du récit, c'est le document pilote essentiel du projet à partir duquel va pouvoir s'organiser tout le travail de réalisation proprement dit. Mais ce n'est ni le seul ni le dernier document de synthèse servant à contrôler le développement du projet. Le scénario fera lui-même l'objet d'une réélaboration sous forme scripturale (le «séquencier») et surtout sous forme graphique (le «découpage» et le «storyboard »).

\section{Exogenèse et endogenèse scénaristiques}

La rédaction d'un scénario original relève bien entendu d'une démarche endogénétique où le scénariste invente une fiction narrative en favorisant autant qu'il est possible, dans son écriture, la dimension visuelle des représentations par lesquelles il raconte son histoire; mais, comme en littérature, il n'est pas rare qu'une certaine dose d'exogenèse soit originairement présente dans son travail qui peut trouver sa source dans un texte littéraire, un film, ou un essai. Au cours d'une interview publiée par Spécial en janvier 1979, Hergé ne faisait pas mystère des œuvres dont il s'était directement inspiré :

Tintin au Pays des Soviets, c'était au départ d'un livre de Joseph Douillet : Moscou sans voiles. Pour Tintin en Amérique, je me suis inspiré des Scènes de la vie future de Georges Duhamel. Le Lotus bleu s'inscrit dans la foulée de La Condition humaine d'André Malraux. Les Cigares du pharaon, c'était une sorte de digest de l'œuvre d'Henri de Monfreid, Les Secrets de la mer Rouge, La Croisière du haschich, etc.

Ici comme ailleurs, à l'exception des cas où l'auteur s'amuse à citer ses sources ou à y faire délibérément allusion, l'emprunt initial a pour destin de disparaître sans laisser la moindre trace dans l'œuvre finale, à la faveur des processus d'intégration par lesquels la genèse scriptographique convertit ses matériaux exogénétiques en matière endogénétique : le style

5. Interview pour La Libre Belgique, 30 décembre 1975. 


\begin{tabular}{|c|c|c|c|}
\hline $\begin{array}{c}\text { PHASES } \\
\text { PROCESSUS }\end{array}$ & $\begin{array}{l}\text { FONCTIONS } \\
\text { OPÉRATOIRES }\end{array}$ & $\begin{array}{l}\text { GENESE SCRIPTURALE } \\
\text { (scénariste) }\end{array}$ & $\begin{array}{c}\text { GENĖE GRAPHIQUE } \\
\text { (dessinateur, équipe graphique) }\end{array}$ \\
\hline $\begin{array}{l}\text { Exogenèse } \\
\text { provisionnelle }\end{array}$ & $\begin{array}{l}\text { Orientation préliminaire } \\
\text { Prospection } \\
\text { Définition de l'organisation } \\
\text { du travail (collaboratif } \\
\text { ou non) }\end{array}$ & $\begin{array}{l}\text { Exploration des projets antérieurs } \\
\text { non aboutis, carnets de notes, d'idées, } \\
\text { de voyage, faits divers, etc. } \\
\text { Recherches et accumulation } \\
\text { d'informations autour de thématiques } \\
\text { préférentielles, en dialogue avec } \\
\text { le dessinateur } \\
\text { Textes littéraires à adapter ou à utiliser }\end{array}$ & $\begin{array}{l}\text { Retour sur d'anciens carnets de } \\
\text { projets et de croquis, choses vues, } \\
\text { photos, films, récits de rêves } \\
\text { Recherches et accumulation } \\
\text { d'informations autour de } \\
\text { thématiques préférentielles, } \\
\text { en dialogue avec le scénariste } \\
\text { Références visuelles }\end{array}$ \\
\hline $\begin{array}{l}\text { Endogenèse } \\
\text { exploratoire }\end{array}$ & $\begin{array}{l}\text { Définition du statut du projet } \\
\text { - nouvelle recherche libre } \\
\text { - nouvel épisode d'une } \\
\text { série déjà publiée } \\
\text { - nouvelle série } \\
\text { Investigation } \\
\text { Délibération } \\
\text { Choix du cadre éditorial : } \\
\text { périodique, album }\end{array}$ & $\begin{array}{l}\text { Recherche ou approfondissement } \\
\text { du thème narratif choisi pour le récit } \\
\text { Hypothèse : nouveau récit, nouvel } \\
\text { épisode d'une série, nouvelle série } \\
\text { Dialogue avec le dessinateur } \\
\text { (figurabilité narrative) } \\
\text { Prescriptions de commande } \\
\text { recommandations de l'éditeur }\end{array}$ & $\begin{array}{l}\text { Ébauches de nouveaux composants } \\
\text { (personnages, situations, milieux) } \\
\text { ou actualisation de composants } \\
\text { déjà élaborés pour des œuvres } \\
\text { antérieures } \\
\text { Dialogue avec le scénariste } \\
\text { (narrativité figurale) } \\
\text { Prescriptions de commande, } \\
\text { recommandations de l'éditeur }\end{array}$ \\
\hline $\begin{array}{l}\text { Exploration } \\
\text { Exogenèse }\end{array}$ & $\begin{array}{l}\text { Documentation exploratoire } \\
\text { Enquête provisionnelle }\end{array}$ & $\begin{array}{l}\text { Notes sur faits divers, rêves, } \\
\text { autobiographie, coupures de presse } \\
\text { Recherche de textes et d'informations } \\
\text { historiques à valeur heuristique } \\
\text { Notes sur l'adaptation possible } \\
\text { d'une œuvre préexistante (film, texte } \\
\text { littéraire, document) }\end{array}$ & $\begin{array}{l}\text { Nouveaux repérages, esquisses } \\
\text { exploratoires et croquis divers }\end{array}$ \\
\hline $\begin{array}{l}\text { Endogenèse } \\
\text { scriptographique }\end{array}$ & $\begin{array}{l}\text { Sélection d'une thématique } \\
\text { Préfiguration du projet } \\
\text { Ébauche d'une } \\
\text { syntagmatique du récit }\end{array}$ & $\begin{array}{l}\text { Notation d'un premier } \\
\text { canevas sommaire } \\
\text { ou } \\
\text { schéma de scénario }\end{array}$ & $\begin{array}{l}\text { Création graphique des } \\
\text { personnages } \\
\text { Recherche des décors et lieux } \\
\text { d'implantation du récit }\end{array}$ \\
\hline INITIALISATION & Proposition narrative & Rédaction du synopsis & \\
\hline $\begin{array}{l}\text { Exogenèse } \\
\text { préparatoire }\end{array}$ & $\begin{array}{l}\text { Documentation générale } \\
\text { préliminaire }\end{array}$ & $\begin{array}{l}\text { Recherche des textes et des } \\
\text { informations techniques ou } \\
\text { historiques utiles à la rédaction } \\
\text { du scénario } \\
\text { Sélection du texte à adapter }\end{array}$ & $\begin{array}{l}\text { Recherches de sources ou de } \\
\text { référents iconographiques } \\
\text { (gravures, photos, vidéos, films, } \\
\text { BD) en rapport avec le scénario }\end{array}$ \\
\hline $\begin{array}{l}\text { Endogenèse } \\
\text { scénaristique }\end{array}$ & Élaboration du récit & $\begin{array}{l}\text { Rédaction du scénario original } \\
\text { ou du scénario d'adaptation }\end{array}$ & $\begin{array}{l}\text { Croquis et esquisses des } \\
\text { personnages en situation, des lieux, } \\
\text { des univers de référence } \\
\text { Définition d'une charte générale de } \\
\text { mise en page : nombre de vignettes } \\
\text { par bande, nombre de bandes par } \\
\text { planche, nombre de planches }\end{array}$ \\
\hline $\begin{array}{l}\text { Endogenèse } \\
\text { organisationnelle }\end{array}$ & $\begin{array}{l}\text { Programmation } \\
\text { Planification et répartition } \\
\text { des tâches entre les } \\
\text { collaborateurs }\end{array}$ & $\begin{array}{l}\text { Présentation du préprojet } \\
\text { scénaristique et négociations avec } \\
\text { l'éditeur ou le rédacteur en chef } \\
\text { du périodique } \\
\text { Calendrier des opérations d'écriture }\end{array}$ & $\begin{array}{l}\text { Présentation du préprojet graphique } \\
\text { et négociations avec l'éditeur ou } \\
\text { le rédacteur en chef du périodique } \\
\text { Calendrier des opérations graphiques } \\
\text { Mobilisation de l'équipe graphique }\end{array}$ \\
\hline
\end{tabular}

Phases 1 et 2 : exploration, initialisation 


\section{Phase 3 : réalisation scriptographique}

du dessin joint à l'originalité de l'histoire et des dialogues finissent par effacer l'extériorité de la source première d'inspiration, au même titre que le recours technique à la photo d'un lieu finira par devenir insoupçonnable dans le crayonné final qui l'aura entièrement homogénéisé avec les autres formes graphiques.

En partant du scénario écrit sur lequel dessinateur et scénariste sont tombés d'accord, le projet peut entrer dans sa phase d'exécution proprement dite qui va consister à réaliser le programme en transposant l'histoire du stade écrit au stade scriptographique.

\section{Structuration textuelle : le séquencier}

Conformément au sens de ce terme dans le vocabulaire du cinéma, le «séquencier» n'est pas un développement ou un approfondissement du scénario mais au contraire une réduction du récit à la pure séquentialisation de ses éléments. Le but du séquencier est de présenter le récit sous la forme d'une stricte description des scènes qui le composent, sans mention des dialogues, en se concentrant sur les étapes de la narration, les principaux rebondissements de l'histoire et la structure dramatique du récit. Il ne contient aucun dialogue. La structure de l'histoire y est établie de la manière la plus rigoureuse possible. La pagination du futur album est présentée. Les séquences sont définies, délimitées et classées dans leur ordre définitif de succession, leurs contenus ne faisant l'objet que d'une récapitulation limitée à quelques phrases. Le séquencier clarifie le scénario en offrant un cadre simplifié - un pur fil conducteur, un «film d'Ariane» - qui va aider le dessinateur à procéder au travail essentiel du « découpage».

\section{Structuration graphique : du découpage au storyboard}

Du point de vue graphique, la phase de réalisation est encadrée par deux grandes étapes de structuration : le découpage et le storyboard. Le découpage intervient au début de la phase de réalisation et constitue le processus de conception graphique majeur sur lequel vont reposer la structure visuelle du récit et toute l'exécution des dessins. Le storyboard intervient au contraire à la fin de la phase de réalisation comme une sorte de bilan graphique du travail réalisé, dans le but de préparer la finalisation prééditoriale de l'œuvre.

Hergé insiste sur l'importance fondamentale et sur les difficultés du «découpage» qui représente une étape essentielle dans le processus d'élaboration scriptographique du récit :

Une fois que j'ai le fil conducteur, commence le plus gros travail : le découpage. C'est comme pour le cinéma, avec cette contrainte supplémentaire qui consiste à conclure chaque page par un suspense ou une surprise. C'est un travail de bénédictin... un vrai casse-tête chinois ! [...] Par ce découpage, je m'efforce de raconter l'histoire, partie par partie, en insérant chacune d'entre elles dans le cadre d'une page, en plaçant les gags, en situant le contenu des images. Je fais quelquefois dix, vingt essais, pour une même page 6 .

Pour sa part Hergé travaille pour son découpage au crayon ou au stylo-bille, sans chercher à faire dans l'élégance, sur des feuillets de format moyen et de papier ordinaire (souvent

6. Interview pour La Libre Belgique, 30 décembre 1975. 


\begin{tabular}{|c|c|c|c|}
\hline $\begin{array}{c}\text { PHASES } \\
\text { PROCESSUS }\end{array}$ & $\begin{array}{l}\text { FONCTIONS } \\
\text { OPÉRATOIRES }\end{array}$ & $\begin{array}{l}\text { GENÈSE SCRIPTURALE } \\
\text { (scénariste) }\end{array}$ & $\begin{array}{c}\text { GENÈSE GRAPHIQUE } \\
\text { (dessinateur, équipe graphique) }\end{array}$ \\
\hline $\begin{array}{l}\text { Endogenèse } \\
\text { textuelle }\end{array}$ & $\begin{array}{l}\text { Composition } \\
\text { Articulation du récit, } \\
\text { planche par planche }\end{array}$ & Rédaction du séquencier & \\
\hline $\begin{array}{l}\text { Endogenèse } \\
\text { graphique }\end{array}$ & $\begin{array}{l}\text { Structuration } \\
\text { 1. planche par planche } \\
\text { 2. de l'ensemble des } \\
\text { planches, de l'incipit } \\
\text { à l'explicit }\end{array}$ & $\begin{array}{l}\text { Distribution du récit en syntagmes } \\
\text { narratifs consécutifs (séquences } \\
\text { contiguës de plusieurs pages), unités } \\
\text { synoptiques (double page, page) } \\
\text { et éléments constitutifs de l'unité } \\
\text { synoptique (bandes et cases ou } \\
\text { vignettes) }\end{array}$ & $\begin{array}{l}\text { Mise en page } \\
\text { Découpage : première } \\
\text { interprétation graphique intégrale } \\
\text { du scénario. Préfiguration des } \\
\text { cases, des bandes, des pages } \\
\text { constituant les futures planches, } \\
\text { des séquences de pages-planches } \\
\text { Storyboard prospectif }\end{array}$ \\
\hline $\begin{array}{l}\text { Exogenèse } \\
\text { scriptographique }\end{array}$ & $\begin{array}{l}\text { Documentation secondaire } \\
\text { spécifiée }\end{array}$ & $\begin{array}{l}\text { Repérages documentaires, notes } \\
\text { de repérages, noms de lieux, } \\
\text { cartographies }\end{array}$ & $\begin{array}{l}\text { Recherches iconographiques } \\
\text { Repérages : croquis, dessins } \\
\text { sur le motif, schémas, plans. } \\
\text { Réalisation des calques ou dessins } \\
\text { documentaires au trait (lieux, } \\
\text { objets) à partir de photos }\end{array}$ \\
\hline RÉALISATION & Exécution graphique & & $\begin{array}{l}\text { Rough. Premiers crayonnés } \\
\text { Premier jet des planches, études de } \\
\text { détail et calques correctifs. Mise en } \\
\text { place des bulles et des appendices. } \\
\text { Figuration des emanata, } \\
\text { idéogrammes }\end{array}$ \\
\hline $\begin{array}{l}\text { Endogenèse } \\
\text { textuelle }\end{array}$ & $\begin{array}{l}\text { Écriture } \\
\text { Textualisation }\end{array}$ & $\begin{array}{l}\text { Rédaction des textes (dialogue, } \\
\text { monologue, commentaire) à intégrer } \\
\text { dans les bulles et les cartouches des } \\
\text { récitatifs }\end{array}$ & $\begin{array}{l}\text { Première mise en place des textes: } \\
\text { bulles, appendices, cartouches } \\
\text { de récitatifs }\end{array}$ \\
\hline $\begin{array}{l}\text { Endogenèse } \\
\text { graphique }\end{array}$ & Figuration narrative & & $\begin{array}{l}\text { Crayonnés de travail (planches, } \\
\text { bandes, vignette) centrés sur les } \\
\text { personnages et le récit (rough). } \\
\text { Mise en attente des figurations } \\
\text { documentées }\end{array}$ \\
\hline $\begin{array}{l}\text { Exogenèse } \\
\text { graphique }\end{array}$ & $\begin{array}{l}\text { Enrichissement } \\
\text { documentaire }\end{array}$ & & $\begin{array}{l}\text { Intégration des calques ou dessins } \\
\text { documentaires aux crayonnés } \\
\text { de travail }\end{array}$ \\
\hline $\begin{array}{l}\text { Endogenèse } \\
\text { graphique }\end{array}$ & Optimisation graphique & & $\begin{array}{l}\text { Sélection des meilleurs crayonnés } \\
\text { de travail et calques. Report et mise } \\
\text { au net (Hergé : calque) des tracés } \\
\text { sélectionnés comme prédéfinitifs }\end{array}$ \\
\hline $\begin{array}{l}\text { Endogenèse } \\
\text { scriptographique }\end{array}$ & $\begin{array}{l}\text { Structuration graphique } \\
\text { définitive }\end{array}$ & Essais de titres & $\begin{array}{l}\text { Résumé détaillé de l'enchaînement } \\
\text { des planches abouties : } \\
\text { Storyboard récapitulatif }\end{array}$ \\
\hline
\end{tabular}

Phase 3 : réalisation scriptographique 
du papier standard de machine à écrire $21 \times 27 \mathrm{~cm}$ ). La mise en page des cases, des bandes et des pages s'organise progressivement, page par page, sous la forme d'ébauches rudimentaires comportant des graphismes sommaires et une transcription manuscrite du dialogue contenu dans le scénario. Même si les tracés sont griffonnés plus souvent que vraiment dessinés, texte et images sont immédiatement considérés comme un tout, le traitement spatial du texte dans les cases faisant partie intégrante du travail de structuration et de séquentialisation sur lequel va reposer l'exécution des crayonnés.

Le découpage est paradoxal : il ressemble à une sorte de montage qui aurait lieu avant la prise de vue. Son rôle est crucial : c'est le moment où le dessinateur va devoir décider ce qui sera retenu et rendu visible ou non de chaque scène prévue dans le scénario, en sélectionnant les moments les plus visuels, les points de vue et les cadrages les plus efficaces, les vecteurs de sens les plus probants, les lignes de dialogues les plus percutantes.

Les crayonnés réalisés ensuite pourront en partie remettre en cause certains choix, mais sur l'essentiel les contenus visuels et les rythmes devront avoir été mis en place. Il s'agit d'une étape génétique fondamentale dont Hergé mentionne la complexité et l'enjeu essentiel : il s'agit d'ordonner la totalité du récit en une suite finie de planches, en respectant exactement le nombre de pages prescrites et en tenant compte de l'importance des scansions par fin de page impaire (page-turning), de l'exigence des pics de suspense (cliffhangers) et de la distribution des «gags » qui ne peuvent valoir pour eux-mêmes mais doivent au contraire accompagner le mouvement narratif et soutenir le rythme de l'action. Pour un album de soixante-deux pages dont certaines pourront quelquefois avoir exigé «dix, vingt essais», combien le découpage final représente-t-il d'ébauches? Plusieurs centaines.

Le storyboard en principe est d'une autre nature : simple mise au net sommaire des images (sans texte), il peut apparaître, soit immédiatement après le découpage, comme une sorte de résumé prospectif des cases et des planches à exécuter, soit, le plus souvent, après la réalisation des crayonnés comme une sorte de bilan récapitulatif servant à préparer la phase de finalisation. Dans l'un et l'autre cas, le storyboard donne au dessinateur le moyen de visionner chaque planche de son récit comme une succession de plans de cinéma en contrôlant la variation et la pertinence des types de plans et des angles de vue choisis, la cohérence visuelle des séquences et du montage, la distribution et l'intelligibilité des ellipses, bref l'ensemble du traitement visuel de la narration.

\section{Exécution graphique et documentation spécifiée}

À partir des prescriptions visuelles que le découpage fixe pour chaque planche et chaque case, le dessinateur peut se lancer, avec son équipe graphique, dans la réalisation des crayonnés : les esquisses de plus en plus précises par lesquelles les séquences de planches pourront évoluer jusqu'à leur forme quasi définitive. C'est le cœur de la genèse graphique, comme la phase rédactionnelle du «brouillon» peut en littérature être considérée comme le cœur de la genèse avant-textuelle : le moment où les dessins passent du croquis sommaire à l'esquisse avancée puis à la mise au net, comme les phrases du brouillon passent de la notation télégraphique à une véritable formulation lexicale et syntaxique contrôlée par le style de l'écrivain, moyennant un nombre parfois impressionnant de ratures et de versions successives du même passage. Le dessinateur suit de près les indications de son découpage (et éventuellement de son storyboard) mais peut se risquer à restructurer les cases de la planche 
en faisant varier les plans, ou en osant un nouveau point de vue plus efficace qui implique un changement de cadrage.

Le trait de détail (expression d'une physionomie, posture dynamique, etc.) peut aussi l'amener à chercher une nouvelle forme plus expressive qui le contraint à de nombreux repentirs pour la même case, y compris jusqu'à remettre en cause la dimension de cette case et sa place dans la planche. Les prescriptions du découpage ne sont donc pas intangibles : la structure de la planche peut à ce stade être sensiblement modifiée, des cases inversées ou agrandies, ou encore supprimées. Dans Le Musée imaginaire de Tintin, Hergé revient sur cette phase de réalisation des crayonnés, particulièrement intense :

C'est la grande bagarre : je gomme, je rature. Je jure aussi. Mais c'est également la grande liberté.

Même si le cadrage n'est pas respecté, ça n'a pas d'importance : il faut que le mouvement y soit7.

La question du «mouvement» est au centre du travail des crayonnés. Le dessinateur doit à chaque instant faire des choix et, considérant les traces de ses arbitrages dans les archives, si le généticien déplore l'usage délétère de la gomme, il se réjouit des semi-effacements qui laissent lire le tracé primitif, des ratures proprement dites et des reprises valant comme variantes. Une bonne part de ces métamorphoses n'a pas d'autre but que d'optimiser la clarté et l'énergie cinétique des tracés.

Et c'est pourquoi, contrairement à ce que l'on pourrait croire, cette optimisation ne consiste pas à parfaire le dessin jusqu'à ce qu'il atteigne l'illusion hyperréaliste de la photographie, mais au contraire ne s'obtient qu'en maintenant dans la forme graphique la plus achevée ce coefficient d'incertitude, de mobilité et de non finito qui appartient en propre à l'esquisse ou même au croquis pris sur le motif. De même que le texte littéraire le plus abouti ne vit que par la mémoire des ratures qui le hantent, en préservant dans sa forme une part d'indécidable, de même le dessin le mieux parachevé est celui qui reste habité par le démon de sa genèse : une infime instabilité, un caprice graphique, la menace d'un déséquilibre ou d'une précarité qui lui vient du geste même de son créateur mais qui va se traduire par un dynamisme secret installé au cœur de la représentation. L'art du crayonné est de conserver le style de l'esquisse dans la forme graphique finalisée.

C'est la vigueur même du rythme narratif qui s'y joue de manière fractale à toutes les échelles de la réalisation graphique : à l'échelle synoptique de la page, à l'échelle séquentielle de la bande, mais aussi à l'échelle minimale de la case et des tracés qui l'animent. Faire que l'œil du lecteur soit attiré par la justesse du détail et dans le même temps soit déjà mobilisé pour passer à l'image suivante est une performance qui suppose un investissement presque physique du dessinateur dans son graphisme. De même que Flaubert, imaginant une scène de roman, ne pouvait la transposer en prose qu'en la mimant physiquement dans son cabinet de travail et finissait par en tester l'exactitude musicale, oralement, en faisant passer ses phrases au «gueuloir», de même Hergé ne parvient à figurer le mouvement de ses personnages qu'en

7. Hergé, Le Musée imaginaire de Tintin, Paris, Casterman, 1979, p. 17. Dans un entretien radiophonique donné la même année, Hergé précise : «C'est à ce stade que j'utilise toute mon énergie. Je dessine furieusement. Rageusement. Je gomme... Je rature... Je remplis... Je surcharge... Je m'acharne... Je jure... J'esquisse d'autres certitudes... Il m'arrive même parfois, qu'à force de revenir sur une attitude, je perce le papier, tout occupé que je suis à donner le maximum d'intensité à l'expression de mes mouvements. » 
ressentant leur dynamique de l'intérieur et, le cas échéant, en se prenant lui-même comme modèle des postures qu'il dessine.

Donner du mouvement est extrêmement fatigant : il faut le sentir chaque fois. [...] J'utilise des miroirs pour étudier des poses. Les plus difficiles sont les poses statiques qu'il s'agit de rendre comme telles, sans figer toutefois le cours de l'action (ibid.).

Si le recours à l'autoportrait peut être considéré comme relevant d'une endogenèse radicale, il est clair que le stade des crayonnés est aussi le moment génétique où le dessin s'enrichit de toutes les recherches exogénétiques qui ont été menées en amont pour lui assurer (sur le rendu des décors, lieux, objets, costumes, etc.) un véritable effet de réel référentiel. Dans le dispositif mis en place par Hergé, c'est la contribution de l'équipe graphique. Une réserve a été ménagée dans les cases qui doivent recevoir le dessin exogénétique et, le reste de l'image étant en place, le dessinateur procède à l'insertion des graphismes documentaires, souvent réalisés sur calques à partir de photos : gros plan d'une carrosserie de voiture, profilé d'un avion, panorama, façade d'hôtel, mobilier d'un salon, etc.

À la différence des documentations générales très diversifiées réunies au cours des phases initiales ( 1 et 2) pour élaborer le scénario et nourrir l'imaginaire des auteurs, l'exogenèse de la phase 3 , commandée par le découpage, est spécifiée à la fois en termes de contenu et en termes de cadrage. Comme en littérature, ces campagnes de documentations exogénétiques ciblées (enquêtes, repérages et croquis sur place, relevés, photos) peuvent avoir pour but de lester le dessin d'une crédibilité réaliste sans pour autant se laisser embarrasser par l'exactitude technique. Si un détail véridique gêne le dessinateur, il en fera joyeusement l'économie en sacrifiant le réalisme aux nécessités de son langage graphique.

C'est ainsi qu'Hergé s'est souvent octroyé des arrangements avec la vérité référentielle de son exogenèse, tout en gardant le bénéfice d'une authenticité entière et même prophétique. C'est le cas, par exemple, dans On a marché sur la lune, pour ses personnages en tenue de cosmonautes qui ont rétrospectivement frappé les esprits lorsque l'opération Apollo de juillet 1969 a donné à voir Neil Armstrong revêtu de son casque et de sa combinaison, arpentant le sol lunaire :

On s'est étonné, une fois les Américains sur la Lune, de voir qu'ils portaient le casque comme Tintin. Je n'ai pas inventé ce casque : je savais qu'il était nécessaire [...]. Mais je l'ai fait transparent, à l'inverse des casques américains qui ne le sont que de face, pour qu'on puisse identifier mes héros, même de dos [...] il serait impossible, sous un casque opaque, de distinguer Tintin du capitaine Haddock ${ }^{8}$.

Toutes ces opérations endo- et exogénétiques se traduisent, pendant la phase d'exécution des crayonnés, par une abondance d'esquisses mais également par une multiplicité de dessins aboutis qui peuvent prétendre à une réelle concurrence : une profusion qui se solde finalement par l'obligation de trancher entre plusieurs variantes possibles. Selon les dessinateurs, à ce stade de décision, les méthodes peuvent être très contrastées, lorsqu'il s'agit de donner forme au crayonné définitif ou prédéfinitif. Certains choisissent de reporter directement les tracés les plus pertinents sur ce qui constituera leur planche définitive; d'autres, plus circonspects,

8. Hergé, interview dans La Libre Belgique, 6 février 1970. 
comme Hergé, préfèrent passer par le calque pour opérer une sélection préalable, en se réservant la faculté de comparer plusieurs hypothèses de montage et de cadrage des meilleurs tracés :

Je vais prendre un calque de tous les crayonnés. Parmi tous ces traits [...] je vais choisir celui qui me paraît le meilleur [...] le plus souple et le plus expressif, le plus clair et aussi le plus simple, celui qui exprime au maximum le mouvement [...] Cette façon de procéder par calque me permet également de cadrer les dessins de façon plus précise (ibid.).

Enfin, cette phase de réalisation des crayonnés n'est pas exclusivement réservée à la dimension graphique de la genèse : tandis que le dessinateur exécute les planches, le scénariste peaufine les récitatifs et adapte les dialogues pour que les répliques tiennent dans les bulles, que les bulles tiennent dans les cases sans menacer l'équilibre du dessin, et aussi pour que le rythme du dialogue participe du mouvement général des images, en s'harmonisant aux temps forts de la narration, aux gags, aux pics de suspense et au fameux page-turning qui introduit nécessairement une solution de continuité dans la lecture.

Mais c'est aussi dans cette phase d'exécution scriptographique que se trouve souvent posée la question du titre de l'œuvre; et les archives des crayonnés comprennent fréquemment, dans les essais portant sur la page d'incipit ou sur la page de couverture, des fragments écrits qui donnent à voir, comme dans les manuscrits d'écrivain, la naissance de l'intitulé de l'œuvre, avec des tentatives de formulation parfois très éloignées du titre que l'album rendra célèbre. Pour l'album paru en 1960 qui sera finalement intitulé Tintin au Tibet, un crayonné préparatoire de la planche 1 (parue dans le Journal de Tintin $\mathrm{n}^{\circ} 38$ en 1958) porte pour titre encadré en haut du feuillet "LE MUSEAU DE LA VACHE», proposition sélectionnée dans une liste écrite au crayon juste au-dessus, en haut de page :

DE L'ÂNE

LE MUSEAU DE LA VACHE..

LE MUSEAU DE L'OURS

DU YACK...

Bien que située dans le prolongement naturel du processus de réalisation scriptographique, la phase 4 de finalisation prééditoriale peut être considérée comme une étape particulière dans la mesure où, comme c'est également le cas en littérature, les opérations de genèse se trouvent désormais largement indexées non plus sur la réalisation elle-même (qui est pratiquement achevée) mais sur les procédures de parachèvement technique qui rendront possible la conversion de l'œuvre autographe en un modèle, reproductible en nombre, satisfaisant aux normes de l'impression. C'est au cours de cette finalisation que le document pilote va passer d'un format de travail, souvent de grandes dimensions, à un format conforme aux exigences de la publication. C'est également au cours de cette étape qu'ont lieu les dernières corrections des textes et des dessins, l'encrage des crayonnés, l'inscription des textes définitifs, et la fabrication du «bleu» sur lequel a lieu traditionnellement le coloriage des planches.

Techniquement, on désigne par le terme de «bleu» l'épreuve tirée, au format de parution, sur laquelle le dessin est reproduit dans un ton très pâle (souvent de couleur bleu azuré). La mise en couleurs est réalisée, non sur la planche originale, mais sur cette épreuve, conformément aux indications autographes données par le dessinateur : toutes les institutions

\section{Phase 4 : finalisation prééditoriale}




\begin{tabular}{|c|c|c|c|}
\hline $\begin{array}{c}\text { PHASE } \\
\text { PROCESSUS }\end{array}$ & $\begin{array}{c}\text { FONCTIONS } \\
\text { OPÉRATOIRES } \\
\text { DOCUMENTS }\end{array}$ & $\begin{array}{l}\text { GENÈSE SCRIPTURALE } \\
\text { (scénariste) }\end{array}$ & $\begin{array}{c}\text { GENÈSE GRAPHIQUE } \\
\text { (dessinateur, équipe graphique) }\end{array}$ \\
\hline $\begin{array}{l}\text { Endogenèse } \\
\text { scriptographique }\end{array}$ & Crayonné prédéfinitif & $\begin{array}{l}\text { Mise à jour des textes à intégrer dans } \\
\text { les bulles et les cartouches } \\
\text { Corrections et adaptation } \\
\text { prédéfinitives du texte intégral des } \\
\text { Vignettes }\end{array}$ & $\begin{array}{l}\text { Crayonné prédéfinitif } \\
\text { Intégration au crayon du texte } \\
\text { définitif dans les bulles assorties } \\
\text { de leurs appendices et dans } \\
\text { les cartouches des récitatifs }\end{array}$ \\
\hline $\begin{array}{l}\text { Endogenèse } \\
\text { graphique }\end{array}$ & Crayonné définitif & & $\begin{array}{l}\text { Mise au net ou crayonné final : } \\
\text { transfert intégral des lignes } \\
\text { pertinentes du crayonné sur } \\
\text { la planche définitive ou planche } \\
\text { originale } \\
\text { Mise en page définitive }\end{array}$ \\
\hline $\begin{array}{l}\text { Endogenèse } \\
\text { graphique }\end{array}$ & Encrage & & Encrage du crayonné final \\
\hline $\begin{array}{l}\text { Endogenèse } \\
\text { scriptographique }\end{array}$ & Lettrage & $\begin{array}{l}\text { Correction et finalisation du texte } \\
\text { intégral des bulles et des récitatifs }\end{array}$ & $\begin{array}{l}\text { Inscription (à la main, ou } \\
\text { typographique) des textes dans } \\
\text { les bulles et les cartouches des } \\
\text { récitatifs, par le dessinateur ou } \\
\text { le lettreur (choix des caractères, } \\
\text { forme, épaisseur, disposition) }\end{array}$ \\
\hline $\begin{array}{l}\text { Endogenèse } \\
\text { graphique }\end{array}$ & Colorisation & & $\begin{array}{l}\text { Tirage du bleu } \\
\text { Mise en couleurs } \\
\text { Gouache, encres (sur papier, } \\
\text { sur calque, etc.) } \\
\text { Travail du coloriste sur épreuve } \\
\text { imprimée }\end{array}$ \\
\hline
\end{tabular}

\section{Phase 4 : finalisation prééditoriale}

\begin{tabular}{|c|c|c|c|}
\hline PHASE & $\begin{array}{l}\text { FONCTIONS } \\
\text { OPÉRATOIRES } \\
\text { PROCESSUS }\end{array}$ & $\begin{array}{c}\text { GENÈSE } \\
\text { SCRIPTOGRAPHIQUE } \\
\text { (auteurs : scénariste, dessinateur) }\end{array}$ & $\begin{array}{c}\text { GENÈSE } \\
\text { ÉDITORIALE } \\
\text { (rédacteur en chef, éditeur) }\end{array}$ \\
\hline & \multicolumn{3}{|l|}{ Bon à tirer } \\
\hline & Fabrication & Ultimes corrections sur film & $\begin{array}{l}\text { Planche gravée, Typon, Ozalid, } \\
\text { film pour l'impression de la bande, } \\
\text { de la planche ou de la séquence de } \\
\text { planches prévues pour publication }\end{array}$ \\
\hline \multirow[t]{2}{*}{$\begin{array}{l}\text { Édition en } \\
\text { périodique }\end{array}$} & $\begin{array}{l}\text { Édition } \\
\text { Diffusion }\end{array}$ & & $\begin{array}{l}\text { Première édition de l'œuvre } \\
\text { (bande, planche ou séquence de } \\
\text { planches) en périodique }\end{array}$ \\
\hline & Réédition en périodique & $\begin{array}{l}\text { Validation ou corrections } \\
\text { et remaniements des textes }\end{array}$ & $\begin{array}{l}\text { Réédition de l'œuvre (bande, } \\
\text { planche ou séquence de planches) } \\
\text { à l'identique ou avec remaniements } \\
\text { (colorisation d'une version publiée } \\
\text { antérieurement en noir et blanc) }\end{array}$ \\
\hline
\end{tabular}

\section{Phase 5 : édition en périodique}


publiques ou privées de conservation, comme le Musée de la Bande dessinée à Angoulême, le Centre belge de la bande dessinée à Bruxelles ou le Musée Hergé de Louvain-la-Neuve, possèdent de nombreuses archives de ce type où les bleus sont légendés de prescriptions autographes précisant les résultats attendus (contours, à-plats, transparences, dégradés, etc.) avec pastilles de couleurs en référence.

Une équipe de spécialistes prend en charge ce travail. Selon les techniques utilisées (gouache, encre, pastel, aquarelle, palette numérique), le dessinateur peut donner aux coloristes des indications très détaillées pour préciser ce qu'il souhaite comme rendu final en termes de valeurs, de contrastes, de lumière, de chromatismes, mais il n'en a plus le contrôle direct. Si le résultat ne lui convient pas, il lui faudra faire reprendre le travail de colorisation à zéro. Le problème va se poser à nouveau chez l'imprimeur, au moment de la fabrication : l'impression couleur (les différents passages d'imposition, le traitement des contours, les zones possibles de décalage et de débordement, etc.) constituera encore un risque sensible d'approximation ou d'altération. L'art de la bande dessinée, comme au Japon l'art traditionnel de l'estampe, suppose que le dessinateur entretienne des relations de coopération constante et approfondie avec les coloristes, les graveurs et l'atelier d'imprimerie chargés d'interpréter et de reproduire son œuvre.

\section{En périodique, en album}

L'édition de la version originale d'un album peut être précédée par une (ou plusieurs) édition(s) du récit publié sous forme de feuilleton en périodique. De nombreuses modifications peuvent évidemment avoir lieu entre ces éditions successives, à commencer, pour les BD du XXe siècle, par la mise en couleurs de versions primitivement publiées en noir et blanc et l'adaptation des contenus aux nouvelles donnes du contexte historique.

La genèse ne s'arrête donc pas à la phase de création scriptographique mais se poursuit, sur un autre mode, à travers une véritable génétique éditoriale (ou «génétique de l'imprimé») assez semblable à ce qu'elle est en littérature, qui intègre non seulement les éditions variantes des albums mais également, en amont, les différentes éditions pré-originales parues en périodiques. C'est le cas pour notre corpus de référence, Les Aventures de Tintin, qui sont parues dans les livraisons hebdomadaires du Journal de Tintin puis qui ont été publiées chez Casterman sous forme d'albums. Mais avant de paraître dans le journal éponyme puis en album, plusieurs épisodes de la saga avaient déjà été publiés par Hergé, avant la Seconde Guerre mondiale, dans deux autres périodiques : Le Petit Vingtième (supplément du journal Le Vingtième siècle) et l'hebdomadaire Counrs vaillants.

Deux tableaux sont consacrés ici aux phases 5 (édition en périodique) et 6 (édition en album) : ils ne correspondent pas nécessairement à des moments successifs de la genèse éditoriale puisque certaines genèses, visant directement l'édition d'un album, pourront avoir fait l'impasse de l'édition en périodique, tandis que d'autres (comme chez Hergé) peuvent connaître plusieurs vagues éditoriales dans lesquelles une édition en album, précédée ou non par une édition antérieure en périodique, se trouve suivie par une nouvelle édition en périodique qui prépare elle-même une deuxième édition en album, publiée sous le même titre mais souvent profondément revue et corrigée. 
Relatifs à un moment de la genèse au cours de laquelle l'éditeur devient une instance de décision majeure, les tableaux se rapportant à l'édition ne contiennent pas les mêmes rubriques que pour les phases antérieures. J'ai restructuré les entrées en traitant en une seule et même rubrique "genèse scriptographique» tout ce qui se rapporte à l'action des auteurs, qu'il s'agisse des modifications du texte (scénariste) ou des graphismes (dessinateur) et en ouvrant une nouvelle entrée «genèse éditoriale» dans laquelle se trouvent traitées l'action et la fabrication de documents relevant de la compétence de l'éditeur, qu'il s'agisse (pour l'édition en périodique) du rédacteur en chef, ou (pour l'édition en album) de l'éditeur proprement dit.

Le volume Tintin au pays de l'or noir (initialement intitulé Au pays de l'or noir), quinzième titre des aventures de Tintin, paru sous forme d'album colorisé en décembre 1950, avait été prépublié en noir et blanc puis en couleurs dans Le Petit Vingtième et dans Cœurs vaillants (du 28 septembre 1939 au 9 mai 1940), avant d'être re-prépublié, en couleurs, après guerre, dans le Journal de Tintin, du 28 septembre 1948 au 23 février 1950 (avec une interruption entre juillet et octobre 1949, en raison d'une dépression qui avait empêché Hergé de travailler pendant trois mois). Mais la genèse éditoriale de ce titre ne s'arrête pas là.

La première version de l'album, parue en 1950, deux ans après la fondation d'Israël, situait l'action en Palestine quelques années plus tôt, à l'époque de l'occupation anglaise : le récit mettait en scène l'action des milices armées juives et les attentats de l'Irgoun qui préparaient l'implantation d'un état israélien. Après la guerre des Six Jours de 1967 et l'entrée du conflit israélo-palestinien dans une logique d'affrontement qui allait bientôt se solder par une nouvelle guerre (Kippour, 1973), Hergé décide de procéder à une réédition revue et corrigée de l'album : en 1971, Tintin au pays de l'or noir voit disparaître toute allusion à la question sensible de la Palestine. Désormais, même si «l'or noir» constitue l'enjeu majeur des nouveaux conflits régionaux et mondiaux (choc pétrolier de 1973), l'épisode se déroule dans un pays imaginaire du même Moyen-Orient.

L'univers de la bande dessinée développe donc bien, comme la littérature, l'espace d'une véritable «génétique de l'imprimé», d'autant plus riche à étudier que les relations entre édition en périodique et édition en album peuvent s'avérer plus complexes qu'il n'y paraît au premier regard. Un dernier exemple permettra d'en juger.

Lorsque Hergé décide, après la guerre, de travailler sur les Aventures de Tintin en se donnant deux supports - les albums proprement dits et le nouvel hebdomadaire éponyme - il se trouve immédiatement placé, pour son nouveau récit (Le Temple du soleil) devant une alternative technique de double format qui va vite se solder par l'émergence d'une double genèse.

Comme il est convenu que l'histoire occupera la double page central du Journal de Tintin, le dessinateur n'a pas d'autre choix possible que de développer parallèlement deux versions du récit : une version conforme à la présentation classique des albums (avec des planches composées de quatre bandes par page, et soixante-deux pages au total) et une version spécialement conçue pour le journal, avec une présentation structurée en trois bandes horizontales superposées qui traversent toute la largeur de la double page centrale. Hergé commence par travailler à partir des images déjà produites pour l'album et construit, semaine après semaine, une histoire variante en se livrant à un véritable montage des reproductions 


\begin{tabular}{|c|c|c|c|}
\hline PHASE & $\begin{array}{l}\text { FONCTIONS } \\
\text { OPÉRATOIRES } \\
\text { PROCESSUS }\end{array}$ & $\begin{array}{c}\text { GENÈSE } \\
\text { SCRIPTOGRAPHIQUE } \\
\text { (scénariste, dessinateur) }\end{array}$ & $\begin{array}{l}\text { GENÈSE } \\
\text { ÉDITORIALE } \\
\text { (éditeur) }\end{array}$ \\
\hline & Préparation de l'album & $\begin{array}{l}\text { Validation, remaniement ou refonte } \\
\text { des textes, des images et de la } \\
\text { structure en vue de la publication } \\
\text { en album. Suppressions, ajouts, } \\
\text { substitutions de vignettes } \\
\text { Restructuration et mise en page } \\
\text { définitive des bandes et des planches }\end{array}$ & $\begin{array}{l}\text { Validation et pagination des } \\
\text { planches définitives constituant } \\
\text { l'album }\end{array}$ \\
\hline & Intitulation & $\begin{array}{l}\text { Sélection du titre définitif de l'album } \\
\text { et des textes de première page de } \\
\text { couverture }\end{array}$ & Arbitrage de l'éditeur \\
\hline & Présentation et habillage & $\begin{array}{l}\text { Conception, crayonnés, finalisation et } \\
\text { réalisation graphiques de la maquette } \\
\text { de couverture (première page portant } \\
\text { le titre, dos et quatrième) } \\
\text { Habillage paratextuel et visuel de } \\
\text { l'album (pages de garde, page de titre } \\
\text { intérieure) }\end{array}$ & $\begin{array}{l}\text { Arbitrage de l'éditeur } \\
\text { Charte graphique }\end{array}$ \\
\hline & \multicolumn{3}{|l|}{ Bon à tirer } \\
\hline & Fabrication & & $\begin{array}{l}\text { Planches gravées, Typons, Ozalids, } \\
\text { film pour l'impression de l'album, } \\
\text { impression, reliure }\end{array}$ \\
\hline \multirow[t]{5}{*}{ Édition en album } & $\begin{array}{l}\text { Édition } \\
\text { Diffusion }\end{array}$ & & $\begin{array}{l}\text { Première édition de l'œuvre } \\
\text { en album }\end{array}$ \\
\hline & Réimpressions & & $\begin{array}{l}\text { Réimpressions et rééditions } \\
\text { à l'identique de la première édition } \\
\text { de l'album }\end{array}$ \\
\hline & Rééditions & $\begin{array}{l}\text { Remaniement des textes et des } \\
\text { images pour la réédition de l'album }\end{array}$ & Rééditions variantes de l'album \\
\hline & Traductions & $\begin{array}{l}\text { Remaniement et adaptation des } \\
\text { textes et des images pour la réédition } \\
\text { de l'album dans une autre langue } \\
\text { Insertion des textes traduits dans } \\
\text { les bulles et les cartouches des } \\
\text { commentaires }\end{array}$ & $\begin{array}{l}\text { Éditions et rééditions (à l'identique } \\
\text { ou variantes) de versions traduites } \\
\text { de l'album }\end{array}$ \\
\hline & Rééditions enrichies & $\begin{array}{l}\text { Textes et iconographie } \\
\text { supplémentaires en vue d'éditions } \\
\text { savantes ou enrichies, édition } \\
\text { génétique, éditions «collector» }\end{array}$ & $\begin{array}{l}\text { Éditions de versions enrichies } \\
\text { de l'album }\end{array}$ \\
\hline
\end{tabular}

\section{Phase 6 : édition en album}


qu'il recadre, découpe, réduit, agrandit, ajuste ou même qu'il redessine entièrement, pour les adapter à la grille de l'hebdomadaire.

Mais dans le Journal, Hergé n'est pas tenu à la norme des soixante-deux pages qui ne s'impose qu'au format en album. Au fur et à mesure que cette double genèse se développe, Hergé se trouve de plus en plus fréquemment tenté de laisser libre cours à son imagination en ajoutant, pour l'édition en périodique, de nouvelles vignettes et même de nouvelles séquences. Et c'est ainsi que Le Temple du soleil, originairement conçu comme un album faisant suite à l'histoire des 7 Boules de cristal, va devenir, dans le Journal de Tintin de 1947, une véritable saga comptant une centaine de variantes graphiques ou textuelles et comportant trois cent trente-trois vignettes de plus que n'en comptera l'édition en album du même Temple du soleil lorsqu'il paraîtra en 1949.

Comme on le voit, après une genèse de l' 'avant-bande » qui n'a rien à envier en complexités et en rebondissements à «l'avant-texte» des écrivains et qui, en dépit de sa dimension graphique, y ressemble beaucoup par de multiples homologies structurales, les métamorphoses que connaît la BD imprimée à la faveur de ses diverses publications, s'avèrent aussi nombreuses, imprévisibles et palpitantes que peuvent l'être les transformations souvent remarquables de l'œuvre littéraire au cours de son destin éditorial.

\section{Vers une théorie génétique de la BD}

Voilà donc, essentiellement fondée sur des exemples empruntés à la tradition du XXe siècle, et spécialement à Hergé, une esquisse de typologie générale qui devrait permettre de se représenter avec une certaine clarté la succession chronologique des événements et des processus qui président à la genèse d'une bande dessinée. La terminologie spécifique au genre, souvent dérivée du vocabulaire du cinéma (crayonné, scénario, synopsis, storyboard, etc.) s'y trouve évoquée au fil des tableaux qui synthétisent les différentes phases de la genèse, chaque nouvelle notion étant rapidement définie et surtout mise en relation fonctionnelle avec le contexte génétique immédiat qui la caractérise. L'approche génétique étant principalement une affaire d'exhaustivité des traces et de classement des documents, il importait tout à la fois que soit déclinée, aussi complètement que possible, la diversité des pièces qui constituent le dossier de genèse et que soit précisé, tout aussi clairement, l'ordre chronologique de leur apparition, c'est-à-dire la succession des opérations dont chacune de ces pièces constitue la réalisation en représentant une étape sur l'axe qui mène le projet à sa réalisation finale : schéma, canevas, synopsis, scénario, cases, bandes, planches, découpage, séquencier, storyboard, etc. En fin de volume, le glossaire reprend par ordre alphabétique l'ensemble de ces notions, en y ajoutant un certain nombre d'autres notions (notamment historiques ou relatives à la réception des œuvres) qui n'avaient pas lieu d'être présentes dans la typologie.

Les tableaux typologiques - conçus pour étudier l'histoire de la bande dessinée papier n'intègrent pas les modifications que l'usage des écrans, des tablettes graphiques, des logiciels de dessin assisté et des scanners peut apporter aujourd'hui au travail du dessinateur, mais cet environnement numérique, tout en offrant au dessinateur de nouvelles ressources et une plus grande liberté d'initiative, ne bouleverse pas radicalement l'esprit de son travail ni l'ordre de ses procédures : se trouvent reconduits sous forme digitale toutes les étapes et la plupart des types de documents et des gestes graphiques qui caractérisaient les genèses papier. Quant au désir et au plaisir de la relation physique au support papier, ils n'ont visiblement pas disparu 
pour la plupart des auteurs qui persistent à manier le crayon et la plume, tout en jonglant, bien sûr, avec les virtualités graphiques qu'y ajoute le digital.

Comme en littérature ou en histoire de l'art, l'approche génétique de la bande dessinée fait apparaître ses capacités méthodologiques inégalées lorsqu'il s'agit de localiser, d'identifier et d'interpréter les processus exogénétiques qui sont à l'œuvre dans la création de l'œuvre : beaucoup plus sûrement qu'avec la critique des sources ou l'intertextualité, la plongée dans l'exogenèse du projet permet de mettre à jour, dès les premiers moments de la conception, l'extraordinaire richesse des emprunts et des références (documentaires, stylistiques, formels, historiques, etc.) dont l'auteur finira par faire la matière originale de son ouvre. Cet aspect paraît essentiel pour l'étude d'un genre par nature multidisciplinaire qui se trouve spontanément réceptif à un éventail très large d'influences.

Mais la génétique de la bande dessinée, pour se doter des moyens scientifiques les plus assurés, doit encore élaborer les outils descriptifs et logiques indispensables à une véritable maîtrise des phénomènes scriptographiques qui sont propres au genre : les concepts permettant d'identifier avec précision les différents états de la performance graphique et leur valeur opératoire, mais aussi les concepts capables d'analyser, à l'état naissant, cette sémantique mixte qui combine les ressources du code visuel et du code écrit pour construire la logique d'une discursivité à la fois hybride et iconique mettant en jeu des phénomènes cognitifs aussi puissants et singuliers que mal connus. De ce point de vue, la génétique de la BD peut être considérée aujourd'hui comme un véritable laboratoire théorique où se jouent des avancées scientifiques considérables non seulement pour l'étude du genre lui-même mais pour l'intelligence des processus de lecture hybride qu'il met en jeu.

PIERRE-MARC DE BIASI est directeur de recherche émérite au CNRS (ITEM), artiste plasticien et écrivain. Ses recherches portent sur l'œuvre et les archives de Flaubert, les processus de création, le papier, le lexique contemporain, l'érotisme, l'histoire de l'art et des idées. Derniers ouvrages parus : Gustave Flaubert, une manière spéciale de vivre (Poche, 2011, prix de la Critique de l'Académie française), La Génétique des textes (CNRS Éditions, «Biblis», 2011), La Fabrique du titre (collectif, CNRS Éditions, 2012), Éros aujourd'hui (collectif, Médium, 2016).

pm.debiasi@wanadoo.fr

Site personnel : http://www.pierre-marc-debiasi.com/ 


\section{Pour une génétique de la bande dessinée}

Avec la bande dessinée, la critique génétique aborde un secteur particulièrement actif et significatif de la créativité contemporaine qui se trouve déjà doté d'une richesse archivistique de premier plan. Envisager dans quelles conditions et avec quelle méthode la création d'une bande dessinée peut devenir interprétable constitue donc aujourd'hui une nouvelle priorité pour les études de genèse. En termes de processus créatifs, la bande dessinée emprunte ses moyens et son vocabulaire à plusieurs arts visuels et particulièrement au cinéma, mais en restant avant tout un art du récit qui partage avec les textes littéraires la même finalité éditoriale : aboutir à une publication imprimée. Cette prévalence du modèle éditorial permet de montrer que les grandes phases de la genèse, les fonctions opératoires du travail de création et les principaux processus d'élaboration de l'œuvre s'ordonnent, pour la bande dessinée, autour d'un axe, assez similaire à celui de la genèse littéraire, allant de la préfiguration d'un projet scénaristique à sa réalisation écrite et dessinée, puis de sa finalisation prééditoriale à son édition (en périodique et/ou en album).

By studying comic strips, Genetic criticism approaches a particularly active and significant domain of contemporary creativity that is already endowed with a remarkably rich archive. So a reflection on the conditions and methods whereby a comic strip can become the object of interpretation is a new priority for genetics studies today. As regards creative processes, comic strips borrow their means and vocabulary from several visual arts, especially film, while they remain above all a narrative art that shares with literary texts the same editorial finality: a printed publication. This supremacy of the editorial model shows that the major stages of the genesis, the operative functions of the creative works and main processes of the work's development for the comic strip are organized around an axis, rather like that of a literary genesis, ranging from the prefiguration of a scenario project to its written and drawn materialization, then from its pre-editorial completion to its publication (as a periodical and/or album).

Mit dem Comic nähert sich die Critique Génétique einem besonders aktiven und bedeutenden Sektor der zeitgenössischen Kreativität, der schon mit einem einmaligen Reichtum an Archivmaterial aufwarten kann. Es wird zu einer neuen vorrangigen Zielsetzung genetischer Studien, zu untersuchen, unter welchen Bedingungen und mit welcher Methode die Hervorbringung eines Comics interpretierbar wird. Hinsichtlich der kreativen Prozesse entnimmt der Comic seine (Ausdrucks)mittel und sein Vokabular verschiedenen visuellen Künsten, speziell dem Kino, doch bleibt er dabei in erster Linie eine Kunst des Erzählens, der mit literarischen Texten das editorische Ziel teilt: nämlich in einer gedruckten Form zu erscheinen. Diese Prävalenz des editorischen Modells erlaubt es, die großen Phasen der Genese aufzuzeigen. Die operativen Funktionen des Schaffensprozesses und die wesentlichen Vorgänge der Ausarbeitung des Werkes ordnen sich im Falle des Comicstrips um eine Achse an, die jener der literarischen Genese recht ähnlich ist. Sie geht von Szenenentwürfen bis zu deren geschriebener und gezeichneter Verwirklichung, dann von der prä-editorialen Finalisierung bis zur Edition (in einer Zeitschrift und/oder in einem Album).
Con la historieta, la crítica genética aborda un sector particularmente activo y significativo de la creatividad contemporánea, que cuenta ya con una riqueza archivística de primer orden. Considerar en qué condiciones y con qué método la creación de una historieta puede ser interpretable constituye hoy, para los estudios de génesis, una nueva prioridad. En términos de proceso creativo, la historieta utiliza los medios y el vocabulario de varias artes visuales -en particular, del cine-, sin dejar de ser ante todo un arte del relato, que comparte con los textos literarios la misma finalidad editorial: desembocar en una publicación impresa. Esta prevalencia del modelo editorial permite demostrar que las grandes fases de la génesis, las funciones operatorias del trabajo de creación y los principales procesos de elaboración de la obra se organizan, para la historieta, alrededor de un eje bastante similar al de la génesis literaria, que va, primero, de la prefiguración de un proyecto argumentativo a su realización escrita y dibujada, y luego, de la finalización pre-editorial a su edición (en un periódico o en un libro).

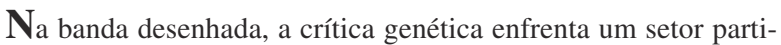
cularmente ativo e significativo da criatividade contemporânea, que já dispõe duma riqueza arquivística de primeira qualidade. Considerar em que condições e com que método a criação de uma banda desenhada pode ser interpretada é hoje prioritário para os estudos de génese. Enquanto processo criativo, a banda desenhada angaria meios e vocabulário junto das artes visuais e especialmente do cinema, mas sem perder o caráter de ser acima de tudo uma arte da narrativa, que compartilha com o texto literário a mesma finalidade editorial, que remata na publicação impressa. Esta prevalência do modelo editorial permite mostrar que as grandes fases genéticas, as funções operativas do trabalho criativo e os principais processos de elaboração da obra são ordenados em torno de um eixo que tem afinidades com a génese literária, desde o projetar do argumento até à sua realização desenhada e escrita, e depois da arte final, pré-editorial, até à edição (em periódico e/ou álbum).

Con il fumetto, la critica genetica affronta un settore particolarmente attivo e significativo della creatività contemporanea, settore che già possiede una ricchezza archivistica di primo piano. Considerare sotto quali condizioni e con quale metodo la creazione di un fumetto diventa interpretabile, costituisce oggi una nuova priorità per la genetica. Se dal punto di vista del processo creativo il fumetto prende in prestito i suoi mezzi e il suo vocabolario da più arti visive - in particolar modo dal cinema -, esso rimane tuttavia un'arte del raccontare, che condivide con i testi letterari una stessa finalità editoriale: la pubblicazione a stampa. Questa prevalenza del modello editoriale permette di dimostrare che le grandi fasi della genesi, le funzioni operatorie del lavoro di creazione e i principali processi di elaborazione dell'opera si dispongono, nel fumetto, lungo un asse piuttosto simile a quello della genesi letteraria; dalla prefigurazione di un progetto di sceneggiatura alla sua realizzazione scritta e disegnata, dalla sua finalizzazione pre-editoriale alla sua edizione (sotto forma di periodico e/o di albo). 